\title{
A novel 2-week wait lung cancer pathway starting with a telephone consultation, with patient satisfaction survey results
}

\author{
Authors: Bavithra Vijayakumar, ${ }^{A}$ Mitra Shahidi, ${ }^{A}$ Raj Thakkar ${ }^{B}$ and Anjani Prasad ${ }^{A}$
}

Nationally, 2-week wait (TWW) referral rates are increasing. This is reflected locally, with no corresponding rise in numbers of new cancers diagnosed. In May 2018, in collaboration with local general practitioners (GPs), we redesigned our pathway to enable speedier diagnosis and reassurance.

First, the referring GP requests computed tomography (CT) of the chest/abdomen and blood tests, at the same time as electronically booking a TWW telephone appointment (OPA) with a respiratory consultant. Initially local GPs were reluctant to book the CT as they did not want the responsibility for chasing and acting on the results, but agreement was reached and the request form now stipulates that the trust are responsible for this.

At the telephone appointment a history is taken, and the CT results and initial investigative plan is explained. At this point those with no cancer can be reassured and discharged. Suitable patients, judged by performance status (PS) and comorbidities, are then sent directly to test or seen face-to-face (F2F) to assess fitness level, or if the patient is felt not to have fully grasped all the issues and needs further explanation.

A satisfaction survey was carried out asking the following questions:

> Did your referring doctor tell you that you had been referred on the cancer pathway?

$>$ Did you know what the phone call was going to be about?

$>$ How did you feel about having a phone call as your first appointment?

> Was the hospital doctor who called you clear about:

$>$ what it was they were investigating?

$>$ what your scans and X-rays showed?

$>$ what the next steps would be?

> Please comment on how we could further improve the service.

The results from the first 111 patients going through the pathway showed high satisfaction with the service; scores were above $93 \%$ in all parameters pertaining to the telephone consultation. Despite initial misgivings about having a telephone conversation as the first contact, our survey shows that the patients appreciated it as it gets them to a diagnosis or reassurance of no cancer diagnosis sooner.

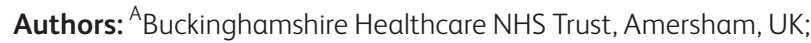
${ }^{B}$ Buckinghamshire Clinical Commissioning Group, UK
After the survey we changed a few processes to improve the quality of the experience.

> There were several patients who had dementia and so were not suitable for a telephone call. After the survey we added an extra question into the referral form for the GP to check that a telephone call was appropriate.

> A significant number of people had expressed a preference for a F2F OPA as their first contact so we now give them the option of coming to a F2F OPA within the same week, rather than pushing on with the call.

We advocate the use of this system. Provided the GP screens the patients as being suitable for a telephone consultation, the patient is given the choice of whether they want to continue with a phone call, and the consultant carrying out the call brings frail/comorbid patients to a F2F OPA before sending off for tests. We have found this to be a safe service with high patient satisfaction.

\section{Conflicts of interest}

None declared. 